\title{
Immunology's dirty little secret
}

Researchers compare the immune systems of wild vs. lab mice

It sounds like the setup to a punch line... an immunologist and a parasitologist walk into a bar... but on this particular evening in 2010, the setup was the beginning of an important collaboration. Over a pint, Eleanor Riley, from the London School of Hygiene and Tropical Medicine, and Mark Viney, from the University of Bristol, UK, hatched a plan to combine forces and study how the immune systems of laboratory mice differ from mice living in the wild. The results, published in Nature Communications, provide a rare and indepth look into immune system function of a wild population of mice, and may provide a critical resource as immunologists confront the difficulties of translating findings from the lab to humans (Nat. Commun. 8, 14811; 2017).

Compared with their feral counterparts, laboratory mice lead a charmed life: they have constant access to food, are safe from predators, and live in clean and hygienic barrier facilities. Wild mice, on the other hand, must struggle to make a living and are constantly exposed to a barrage of microorganisms. Given the differences in their environment, one might expect the immune systems of laboratory and wild mice to differ as well, but it's a question few immunologists have addressed.

"I think if you take most immunologists working with lab mice and press them, they will acknowledge that wild animals are going to be immunologically different," says Viney. "I think you have to press them though... it's not something they would volunteer, since it raises a big concern in the field." But this concern-whether or not clean mice are always the best models to understand and develop treatments for the human immune system-is gaining traction (see Nature 532, 512-516; 2016), despite there being only a small handful

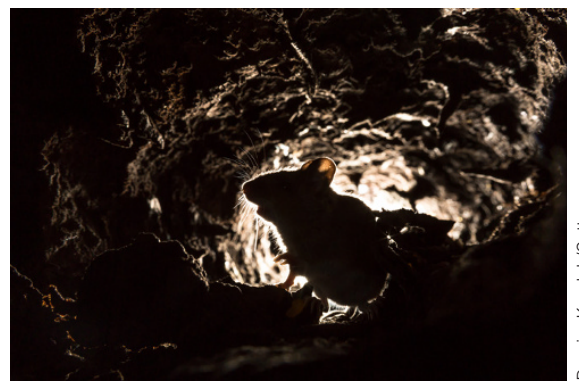

of papers in the literature looking at wild rodent immune function.

Riley and Viney decided the time was right to take a deeper look, and made the most of their complementary expertise: Riley is an immunologist with experience in translational research, and Viney is a parasitologist and field ecologist with experience studying infections in wild rodents. Using wild animals trapped from a variety of sites, from farms near Bristol, in southwest England, to the depths of the London Underground, their team measured the differences between wild and lab mice in several key anatomical, physiological and immune system parameters.

At the whole-animal level, their data suggest that wild mice live hard and die young. $75 \%$ of trapped mice were 12 weeks or younger-as estimated by eye lens massand were significantly smaller in total body mass (typically, about half the size of lab mice used in the study).

Not surprisingly, wild mice carried a significantly higher burden of infection compared to clean lab mice, with upwards of $80-90 \%$ prevalence for infections with a variety of pathogens, including parasitic nematode pinworms (Syphacia spp), mites (Myocoptes musculinus) and several viruses. To counter this inequality, the immune systems of wild mice are much more active, with significantly higher serum levels of IgG and IgE, as well as significantly higher levels of antigen-experienced CD4 + and CD8 + T-cell subsets (effector/effector memory and terminally differentiated cells). The research team also found a previously unidentified myeloid cell population in wild mice, which the group termed hypergranulocytic myeloid cells. Surprisingly, the innate immune responses of wild mice to microbial ligands were similar to-or lower than-those of lab mice, suggesting that in wild mice, immune responses are carefully regulated in order to prevent immunemediated damage.

For Viney, although immune system differences per se were not surprising, the differences ran counter to his intuition from his group's work on rodent infections by the parasitic nematode Strongyloides spp. "If you take lab animals and give them Strongyloides infections, those rats expel the worms and become immune to reinfection after about 4-5 weeks", explains Viney. But Strongyloides infection in wild rats has a prevalence of $\sim 65 \%$, indicating they do not generate an effective immune response to the parasite, the way that lab rats do. "My naive assumption was that wild rodents, with their rough and miserable lives, make rubbish immune responses, but our new results suggest it's completely the opposite; they make enormously strong immune responses...which means we may need to rethink what's going on with respect to parasites."

Riley suspects that their results will prove useful towards understanding the differences in immune responses generated by humans living in different environmental conditions. "On the human side, one of the things we are beginning to see is that if we develop vaccines in Europe or the US and then take those vaccines to Africa, we find that we get very different immune responses," she explains. "So we can see even in humans that the environment helps to calibrate our immune response, and that's exactly what we're seeing in lab vs. wild mice."

Dustin M. Graham 